\title{
RECIRCULATING INDUCTION ACCELERATORS FOR INERTIAL FUSION: PROSPECTS AND STATUS
}

A. Friedman, J.J. Barnard, M.D. Cable, D.A. Callahan, F.J. Deadrick, S. Eylon, ${ }^{+}$T.J. Fessenden, ${ }^{+t}$ D.P. Grote, H.A. Hopkins, V.P. Karpenko, D.L. Judd, ${ }^{++}$H.C. Kirbie, D.B. Longinotti, ${ }^{\text {t+t }}$ S.M. Lund, L.A. Nattrass, M.B. Nelson, M.A. Newton, T.C. Sangster, and W.M. Sharp

Lawrence Livermore National Laboratory, University of California P.O. Box 5508, L-440, Livermore California 94550, USA

\author{
${ }^{+}$Titan Beta \\ Dublin, California 94568, USA \\ ${ }^{+1}$ Lawrence Berkeley Laboratory, University of California \\ Berkeley California 94720, USA \\ RECEIVED \\ DEC 271995 \\ ${ }^{+1}$ EGEG Energy Measurments, Inc. \\ Pleasanton, California 94588, USA
}

This paper was prepared for submittal to the

Proceedings of the International Symposium On Heavy Ion Fusion

Princeton, New Jersey

September 6-9, 1995

September 3, 1995

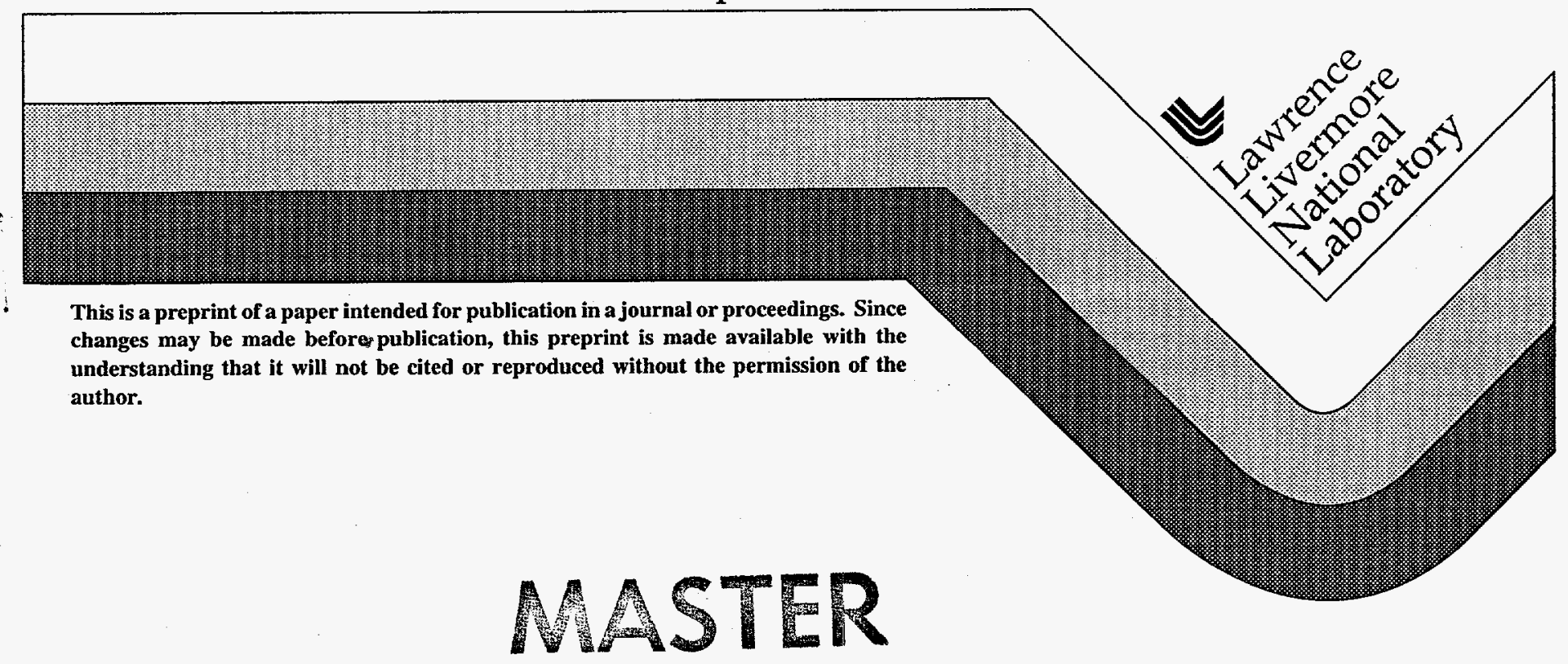




\section{DISCLAIMER}

This document was prepared as an account of worksponsored by an agency of the United States Government. Neither the United States Government nor the University of California nor any of their employees, makes any warranty, express or implied, or assumes any legal liability of responsibility for the accuracy, completeness, or usefulness of any information, apparatus, product, or process disclosed, or represents that its use would not infringe privately owned rights. Reference herein to any specific commercial products, process, or service by trade name, trademark, manufacturer, or otherwise, does not necessarily constitute or imply its endorsement, recommendation, or favoring by the United States Government or the University of California. The views and opinions of authors expressed herein do not necessarily state or reflect those of the United States Government or the University of California, and shall not be used for advertising or product endorsement purposes. 


\section{DISCLAMMER}

Portions of this document may be illegible in electronic image produets. Images are produced from the best available original document. 


\title{
RECIRCULATING INDUCTION ACCELERATORS FOR INERTIAL FUSION: PROSPECTS AND STATUS*
}

\author{
A. Friedman, J. J. Barnard, M. D. Cable, D. A. Callahan, F. J. Deadrick, S. Eylon, \\ T. J. Fessenden, $\ddagger$ D. P. Grote, H. A. Hopkins, ${ }^{\dagger}$ V. P. Karpenko, D. L. Judd, $\ddagger$ H. C. Kirbie, \\ D. B. Longinotti, $\$$ S. M. Lund, L. A. Nattrass, M. B. Nelson, M. A. Newton, \\ T. C. Sangster, and W. M. Sharp \\ Lawrence Livermore National Laboratory, L-440, Livermore CA 94550 USA \\ †Participating Guest \\ łLawrence Berkeley National Laboratory \\ §EG\&G, Inc.
}

\begin{abstract}
The U.S. is developing the physics and technology of induction accelerators for heavy-ion beam-driven inertial fusion. The recirculating induction accelerator repeatedly passes beams through the same set of accelerating and focusing elements, thereby reducing both the length and gradient of the accelerator structure. This promises an attractive driver cost, if the technical challenges associated with recirculation can be met. Point designs for recirculator drivers were developed in a multi-year study by LLNL, LBNL, and FM Technologies, and that work is briefly reviewed here. To validate major elements of the recirculator concept, we are developing a small $(4.5-\mathrm{m}$ diameter) prototype recirculator which will accelerate a space-charge-dominated beam of $\mathrm{K}^{+}$ions through 15 laps, from 80 to $320 \mathrm{keV}$ and from 2 to $8 \mathrm{~mA}$. Transverse beam confinement is effected via permanent-magnet quadrupoles; bending is via electric dipoles. This "Small Recirculator" is being developed in a build-and-test sequence of experiments. An injector, matching section, and linear magnetic channel using seven half-lattice periods of permanent-magnet quadrupole lenses are operational. A prototype recirculator half-lattice period is being fabricated. This paper outlines the research program, and presents initial experimental results.
\end{abstract}

\section{Introduction}

An induction accelerator works by passing the beam through a series of accelerating modules, each of which applies an electromotive force (emf) to the beam as it goes by; effectively, the beam acts as the secondary winding of a series of efficient 
one-turn transformers. Each of these transformers requires a sizable ferromagnetic toroid, or core. The cores must be made large enough to sustain the specified accelerating voltage during the transit of the entire beam.

In the recirculating induction accelerator, or recirculator, the beam is repeatedly passed through the same set of accelerating cores and focusing elements, thereby reducing the length of the accelerator and the number of cores and magnets required. Furthermore, in a recirculator it is not necessary to accelerate the beam quickly to minimize length and cost, as is the case in a linear accelerator, or linac; acceleration can be more gradual, over many laps, and this allows the use of smaller and less expensive cores. This promises a very attractive driver cost if the technical challenges associated with recirculation can be met.

The beam in a recirculator is projected to behave much like that in a long induction linac. A slow addition of beam space charge into a storage ring eventually leads to beam loss because the beam betatron frequencies are gradually shifted until (at the "Laslett tune shift limit") one of them falls into resonance with field errors, which then act on the beam coherently [Laslett, 1]. In contrast, the recirculator avoids beam loss at high current by passing the beam rapidly through the resonances as it is accelerated and compressed. Essentially, the beam is different on every lap, and errors add randomly as they do in a linac. Acceleration rapid enough to avoid resonanant loss is still much more gradual than in an induction linac.

The recirculator concept as it applies to an ICF driver has been described previously [Barnard et. al., 2]. In Section 2 we briefly review the recirculator point designs developed in a multi-year study [Barnard et. al., 3], and comment upon some of the key challenges that will be faced in making the concept a reality.

In Section 3 we describe the design of a small (4.5-m-diam) prototype recirculator, which will accelerate singly charged potassium ions (atomic weight $39 \mathrm{u}$ ) through 15 laps, increasing the ion energy from 80 to $320 \mathrm{keV}$ and the beam current from 2 to $8 \mathrm{~mA}$. Beam confinement and bending are effected with permanent-magnet quadrupoles and electric dipoles, respectively. The dimensionless beam dynamics parameters were chosen to resemble those of a driver; the experiments should serve to validate major elements of the recirculator concept. 
The design is based on scaling laws [Barnard et. al., 4] and on extensive particle and fluid simulations of the behavior of the space charge-dominated beam. In Section 4 we briefly describe some of the modeling tools employed.

The Small Recirculator is being developed in a build-and-test sequence of experiments, the plans for which are described in Section 5. Linear experiments are underway. Near-term plans include studies of space charge-dominated beam transport around a bend. Later we will study insertion and extraction of the beam into and out of the ring, and acceleration with centroid control. The ultimate goal is demonstration of flexible recirculator operation.

An injector and matching section are operational, and we are currently investigating intense-beam transport in a linear magnetic channel. In Section 6 we describe the current status of these experiments and briefly present some initial results.

In Section 7 a few final observations and conclusions are offered.

\section{Recirculators as ICF Drivers}

A multi-year study of recirculators by LLNL, LBNL, and FM Technologies [Barnard et.al., 3] focused on $4 \mathrm{MJ}, 10 \mathrm{GeV}$ drivers accelerating a singly-charged ion of mass 200 . The recirculator drivers considered in the study were four-beam multi-ring designs, in which each ring increased the beam's energy by an order of magnitude over 50 to 100 laps. In contrast with most HIF induction linac concepts [Bangerter, 5], the recirculator designs considered to date use magnetic focusing lenses for transverse confinement, and a constant number of beams throughout. No merging is employed; the injection of a sufficient quantity of ions is effected by a long initial pulse. An artist's conception of a power plant based on such a driver is shown in Figure 1.

Three point designs were considered in the study. The most attractive of these designs was labeled the "C-design" because it represented an attempt to minimize recirculator cost, both by employing more advanced physics and engineering concepts than the other designs and by taking credit for projected decreases in component and materials costs. For example, the C-design employs steady-state superconducting "bias". dipole (bending) fields as part of a combined-function magnet which also provides the quadrupole fields needed for beam confinement. Thus, the time-varying dipole magnet fields sweep from the appropriate $-\Delta B / 2$ to $\Delta B / 2$, thereby reducing the 
stored field energy and power requirement relative to a design which ramped the field from the appropriate initial $B_{0}$ to $B_{0}+\Delta B$.

In the $\mathrm{C}$-design, the injector creates a $200 \mu$ s pulse of ions at $3 \mathrm{MeV}$. The Low Energy Ring (LER) increases the beams' energy to $50 \mathrm{MeV}$, while decreasing the pulse duration to $30 \mu \mathrm{s}$. The Medium Energy Ring (MER) takes the beams to $1 \mathrm{GeV}$ and 2.5 us, while the High Energy Ring (HER) takes them to $10 \mathrm{GeV}$ and 0.25 us. Compression to the final $10 \mathrm{~ns}$ is accomplished after the beams have left the recirculator; a velocity "tilt" (head-to-tail gradient) is imparted in an induction-driven bunching section and the beams shorten in a drift-compression line on their way to the final focus onto the target. The radius of the HER is $314 \mathrm{~m}$, and the final current of each beam in the HER is $400 \mathrm{~A}$. The overall accelerator length is about $3.6 \mathrm{~km}$.

The long initial pulse must be compressed in time by a large overall factor to meet target requirements. This necessitates significant longitudinal (spatial) compression. Space-charge dominated beams behave much like incompressible fluids, and as their length is reduced their cross-section increases correspondingly. In some cases the energy increase in any given ring is limited by the transverse expansion of the beam. The size of the HER is set by the maximum desired bending field at the final energy. Multiple rings are employed because it is desirable to tailor the pulsers and cores in a given ring to the beam characteristics over a limited range of energies, and because the introduction of smaller rings makes it easier to accomodate the increase in beam cross-sectional area due to compression.

The issues which must be addressed for a practical recirculator driver fall into three general categories: precise power handling at high repetition rates, particle loss issues closely related to vacuum requirements, and beam dynamics issues. Control and timing are more complicated than in a linac, as well.

The induction core pulsers in a driver must provide low-noise, flat or triangular $100 \mathrm{kV}, 0.5 \mathrm{kA}$ accelerating pulses with pulse-to-pulse variable format at variable repetition rates of $\sim 100 \mathrm{kHz}$, with a reset pulse of opposite polarity between each accelerating pulse. In addition, additional shaped pulses ("ears") must be provided at the beginning and end of the beams' passage through at least some of the accelerating gaps; these provide the inward impulses which confine the beam longitudinally against its own space charge. These requirements are challenging, but advances in solid state power electronics should make it possible to meet them 

velocities $\left(z, v_{z}\right)$ is at best conserved; such conservation requires smooth, wellcontrolled accelerating pulses. The study did not assume the use of advanced chromatically corrected optics, nor an advanced scenario with large longitudinal acceptance such as a plasma lens/channel transport system. Under the study's assumptions the relative injected voltage jitter (which becomes velocity spread downstream) must be kept $\leqslant 4 \times 10-4$. By comparison, the new ESQ injector at LBNL is achieving a voltage flatness of $1.5 \times 10-3$. Careful implementation of present-day injector pulse-forming techniques may suffice (though the pulse is very long); however active regulation of the injector voltage and/or downstream correction may be necessary. Some of the challenges imposed by the long initial pulse and four-beam nature of the C-Design recirculator may be mitigated by adoption of a 12- or 16-beam layout, at the expense of additional complexity.

The overall path length traversed by the beam in the C-design recirculator is of order $200 \mathrm{~km}$, much longer than that in a linac. Furthermore, and in contrast with a linac, the beam repeatedly visits each section of the beam line. Thus, any material desorbed from the walls as a result of particle loss on one lap is able to affect the beam on subsequent laps-vacuum pumping is ineffective on the inter-lap timescale. The processes accounted for in the design are beam-beam charge exchange, beam stripping by residual gas, and desorption of wall material induced by stray beam ions and ionized gas. The desorption coefficient for heavy ions is believed to be less than unity, except at glancing incidence; this may recommend a series of aperture disks be included in the beam pipe to avoid such impacts. A high vacuum of order $10^{-11}$ to $10^{-}$ 10 Torr is required to minimize beam-gas interactions.

The C-design was constrained to allow $3 \%$ beam loss in the HER. Based on experience with structure activation at LAMPF [FEAC Panel 7, 11], the design is marginal for "hands-on" maintenance of the accelerator, and more detailed study is needed.

"Hybrid" designs (with a recirculator at the low-energy end and a linac at the high-energy end) are also possible and may prove attractive. Such designs may have favorable attributes, including minimization of beam loss at high energy and the potential for incremental increases in total delivered energy in a facility upgrade. This option has not yet been studied. 
The beam-dynamics issues that must be resolved before a recirculating driver can be built include centroid control, longitudinal control, and beam insertion and extraction. A small enough beam emittance must be preserved throughout, in the presence of bend and space-charge forces, so that the beam can eventually be focused onto a small spot. Precise alignment and active beam steering will be required. As described below, beam-dynamics issues can be addressed at reduced scale in a small prototype recirculator.

\section{Design of the Small Recirculator}

LLNL is developing a small prototype ion recirculator in collaboration with LBNL, EG\&G, and Titan-Beta. This Small Recirculator will be assembled and operated as a series of experiments over several years. Figure 2 illustrates the overall physics design of the Small Recirculator and lists some of the elements that must all work together in the Small Recirculator and in a full-scale fusion driver.

In contrast with conventional rf-driven accelerators, the beam in the Small Recirculator experiments is space-charge dominated. That is, the transverse force balance is a near-cancellation of the applied confining force and the beam's selfinduced electrostatic expanding force; thermal pressure plays only a minor role. Effectively, the beam is a non-neutral plasma in nearly laminar flow. The fully operational Small Recirculator will confine and accelerate many more ions than a conventional circular accelerator of this scale and beam energy.

The Small Recirculator will have a circumference of $14.4 \mathrm{~m}$, a $3.5-\mathrm{cm}$ aperture (pipe) radius for the beam-confining (commonly called focusing) and beam-bending elements, and a $72-\mathrm{cm}$ lattice period (segment of the repetitive lattice of focusing and bending elements). The beam will be transversely confined by permanent-magnet quadrupole lenses with a field of $\sim 0.294 \mathrm{~T}$ at the pipe wall, and will be bent with electric dipole deflector plates. These quadrupoles and dipoles will each physically occupy about $30 \%$ of the axial lattice length, and the full recirculator ring will consist of 40 half-lattice periods, including one or two periods using special large-aperture quadrupole magnets through which the beam will be inserted and/or extracted. The fundamental building block is actually the $36-\mathrm{cm}$ half-lattice period, but the polarity of the quadrupole lenses is reversed in each alternate half-lattice period; this provides so-called alternating-gradient or strong focusing as in most modern particle accelerators. 
The $\mathrm{K}^{+}$beam ions will be accelerated from an initial kinetic energy of $80 \mathrm{keV}$ to $320 \mathrm{keV}$ over 15 laps by 34 induction cores (no induction cores will be present in the lattice periods where the beam is inserted and extracted). The initial beam current will be $2 \mathrm{~mA}$, corresponding to a line-charge density of $3.6 \mathrm{nC} / \mathrm{m}$ and a characteristic beam radius of $1.1 \mathrm{~cm}$, and the initial pulse duration will be $4 \mu \mathrm{s}$. After 15 laps of acceleration, the beam current will have increased to $8 \mathrm{~mA}$, the line-charge density will be $7.21 \mathrm{nC} / \mathrm{m}$, the average beam radius will be $1.3 \mathrm{~cm}$, and the pulse duration will be $1 \mu \mathrm{s}$.

Because the quadrupole magnets provide a transverse restoring force to confine the beam, the beam centroid will, if displaced off-axis, oscillate back and forth across the centerline of the beam pipe. Over a full oscillation, the phase of the displacement will sweep through $360^{\circ}$. The initial phase advance of these "betatron oscillations" per lattice period of beam motion will be $\sigma_{0}=78^{\circ}$. Thus a beam-centroid oscillation will require $360^{\circ} / 78^{\circ} \approx 4.6$ lattice periods, or about $4.6 / 20=23 \%$ of the circumference of the ring. Individual particles also execute betatron oscillations back and forth within the confines of the beam, but the frequency of these oscillations is lower than the "undepressed" frequency because the net focusing force is reduced by the repulsive effects of space charge, which (if unopposed) would blow up the beam. Initially, the net effect is a phase advance depressed to $\sigma=16^{\circ}$ by space charge. After 15 laps of acceleration, the phase advances will decrease to $\sigma 0=45^{\circ}$ and $\sigma=12^{\circ}$. These parameters were chosen to resemble those of a driver-scale recirculator, although of course the latter would have many more betatron oscillations per lap.

Because the heavy-ion beam in the Small Recirculator is nonrelativistic and accelerating, obtaining the variable-format accelerating and bending waveforms will be technologically challenging. Those waveforms will require the accurate synthesis of detailed voltage pulses with repetition rates rising from about $40 \mathrm{kHz}$ at the initial beam energy to $90 \mathrm{kHz}$ at the final beam energy [Fessenden et. al., 12, Kirbie et. al., 6, and Newton et. al., 7]. The voltage pulses for the electric dipoles must be correctly ramped in concert with the increasing beam energy. Properly shaped "ear" pulses must be applied at the beginning and end of the main pulses to provide longitudinal confinement, and lap-to-lap variation of the pulse duration and shape must be added to the accelerating waveforms to maintain or decrease the beam length.

To switch the beam into or out of the ring, time-varying dipole (bending) fields must be applied. Transverse confinement of the beam must be carried out during 
insertion or extraction. Our design uses a permanent-magnet quadrupole with an expanded aperture [Lund et. al. , 13]. Figure 3 shows the physics design of the insertion/extraction section. The main ring runs along the lower part of the figure; the insertion line (which brings the beam from the matching section into the ring) comes in from the upper left, and the extraction line runs toward the upper right. The beam trajectory is shown as it will appear during extraction.

Mechanical design of the Small Recirculator was challenging because of the necessity of fitting bending, foctusing, and accelerating elements, as well as provisions for vacuum pumping and beam diagnostics, into each half-lattice period. Figures 4 and 5 show the nearly complete computer-aided design, as rendered by the CAD software.

For more details on the considerations leading to the design of the Small Recirculator, refer to [Barnard et. al., 4].

\section{Recirculator Modeling}

Because the space charge-dominated beams in an induction accelerator are effectively non-neutral plasmas, theoretical and computational modeling of these beams is carried out using techniques related to those used in the accelerator and plasma physics communities. Models used range from simple zero-dimensional codes based on analytically derived scaling relations, through fluid- and momentequation simulations, up to large and elaborate discrete-particle simulations.

The CIRCE code [Sharp et. al., 14] is a multidimensional model that solves an envelope equation (evolving moments such as centroid position and transverse extent) for each of a number (typically a hundred or greater) of transverse beam "slices," each at different longitudinal positions. The longitudinal dynamics of the beam is modeled by evolving the positions and velocities of the slices using fluid equations. CIRCE is used to assess alignment tolerances, accelerating schedules, and steering techniques in linacs and recirculators. It is useful for any application in which the evolution of the detailed internal degrees of freedom of the beam (e.g., emittance growth processes) need not be resolved; at present, beam normalized emittance is assumed constant in CIRCE. The code has played an important role in the development of the beam accelerating and confining waveforms needed for the Small Recirculator. 
Because the beam resides in the accelerator for relatively few plasma oscillation periods, particle-in-cell (PIC) simulation techniques are especially effective and have been invaluable in the design and analysis of experiments and in the prediction of the behavior of future machines. The WARP code includes fully three-dimensional (WARP3d) [Friedman et. al., 15] and axisymmetric (WARPrz) [Callahan et. al., 16] PIC simulation models. The ultimate goal of this code development is effective simulation of present-day experiments and of an HIF driver, from the source through the final focus, with a link from WARP into the codes used to model propagation in the fusion chamber and ultimately into the target design codes.

Emittance growth can result from the nonuniform distribution of beam space charge resulting from the action of centrifugal forces. As revealed in particle simulations using WARP3d [Friedman et. al., 15] and interpreted theoretically [Barnard et. al., 17], growth occurs at changes in the accelerator's curvature where the distribution of beam particles relaxes toward a new equilibrium. A circular recirculator is therefore to be preferred over one with an elongated "racetrack" shape. Since the Small Recirculator is effectively circular (the changes of curvature that occur within a single half-lattice period are too rapid to matter), the only significant changes in curvature occur during insertion and extraction. The electric dipoles also introduce field aberrations. Detailed 3-D simulations show that proper shaping of the dipole plates should render the beam distortion minimal. We have studied the behavior of the beam in the Small Recirculator in some detail using WARP [Grote et. al., 18]. A measurable amount of emittance growth is expected to take place over the 15 laps, mostly in the first two laps as the beam adjusts to the bent lattice of the ring .

\section{Experiment Plans}

Linear experiments now getting under way will measure space chargedominated beam quality after transport through a permanent-magnet quadrupole lattice, characterize the beam before injection, provide a test bed for diagnostic development, and afford a preliminary assessment of the role of electrons in magnetic beam transport (see Fig. 6).

The next experiments will study beam transport around a bend of order $90^{\circ}$ (at first without any accelerating modules). The transition of the beam from a straight transport line into the ring will represent a change of curvature, and will allow us to study the resulting emittance growth. Emittance growth can also result from 
imperfections in the focusing and bending fields; the small imperfections expected in our experiments will be well characterized by theory and measurement. Even over a short bend, detailed intercepting beam diagnostics (using a two-slit apparatus to measure both transverse ion position and velocity) should be able to detect relatively small changes in the distribution of beam particles as a result of the bend. An important goal of these initial experiments will be validation of the computer models and scaling laws used to predict the behavior of linear and recirculating drivers.

Later experiments will study insertion and extraction, acceleration (at first in a partial ring to facilitate measurement of the beam using intercepting diagnostics), beam steering, bunch compression, and fully integrated operation of the recirculator. Preservation of a small emittance will again be the central beam-physics issue to be addressed.

Until the ring is complete it will be possible to use intercepting diagnostics to characterize the beam and to calibrate the nonintercepting diagnostics that will be critical to the successful operation of the full ring. As currently planned, the ring will incorporate one or two extraction sections, so the extracted beam can be diagnosed with detailed intercepting diagnostics once or twice each lap. As with earlier linac experiments at LBNL, excellent shot-to-shot repeatability is anticipated and, so far, observed. The principal nonintercepting diagnostic under development is a segmented capacitive pickup to be located inside the quadrupoles [Deadrick et. al., 19, and Fessenden et. al., 20].The long duration of beam residence in the machine (up to, and possibly exceeding, 300 full lattice periods) will provide a unique opportunity to observe and characterize the longitudinal propagation of space-charge waves along the beam. Such waves will be launched (deliberately or otherwise) by mismatching the applied ear fields. The Small Recirculator will afford the longest beam path length of any near-term HIF research facility, and so will be able to explore issues such as slow thermalization that are important to both recirculating and linear drivers.

\section{Status and Initial Results}

The injector diode [Eylon et. al., 21], matching section, and straight experiment have been fabricated and are now operating; Fig. 6 shows the layout. Fifteen permanent-magnet quadrupoles have been procured; seven are being used in the straight experiment (see Fig. 8). A shorter line will serve as the link from the 
matching section to the ring. As shown in Figs. 4 and 5, the mechanical design of the half-lattice period is nearly complete [Karpenko et. al., 22].

The electrostatic-quadrupole matching section (Fig. 6) gives the circular beam that leaves the diode an elliptical cross section suitable for alternating-gradient transport in the transfer line and the recirculator. A section of the Single Beam Transport Experiment (SBTE) apparatus from LBNL was adapted by EG\&G to serve this function. The potentials applied to the various quadrupole elements to obtain a matched beam were derived using an envelope calculation and range from \pm 1.8 to $\pm 4.0 \mathrm{kV}$. The fifth and seventh elements are intended for minor beam steering rather than for focusing. Insertable Faraday cups are located after the third and ninth elements.

Figure 7 shows the source and diode. A potassium-impregnated zeolite element is heated by an internal filament; the beam passes through the hole visible at the left end of the anode-cathode gap. Figure 8 shows the linear transport section.

Time-resolved measurements of beam properties have been obtained at various locations throughout the matching and magnetic transport sections. The current has been measured using Faraday cups 0.67 and $1.9 \mathrm{~m}$ downstream from the diode source in the matching section and $3.16 \mathrm{~m}$ downstream in the magnetic transport section. An energy analyzer developed at LBNL (consisting of curved electrostatic plates across which various potential differences are placed) was located $1.75 \mathrm{~m}$ downstream from the source. A two-slit scanner was placed at positions 0.2 and $1.6 \mathrm{~m}$ downstream from the source, providing measurements of emittance, beam radius, and beam centroid location.

Figure 9 shows an example trace of current vs. time at the Faraday cup $1.9 \mathrm{~m}$ downstream from the diode source and corresponding results from the 1-D code HINJ [Barnard et. al., 23]; there is close agreement between simulation and experiment. The large current spike at the head of the pulse arises because the rise time of the diode voltage (about $1 \mu \mathrm{s}$ ) is longer than the ideal rise time of $0.48 \mu$ s [Lampel and Tiefenback, 24]. With the longer rise time, particles emitted at the beginning of the pulse have significantly lower energy than particles emitted later, so particle overtaking occurs. A modification of the pulser circuitry to reduce the rise time by a factor of two is planned. The code results are slightly noisier than those from the experiment; this results from a numerical deconvolution of the voltage waveform (to 
account for time lags in the voltage monitor), which introduces noise into the voltage waveform used by the code. Figure 10 shows a measurement of the horizontal normalized emittance at the end of the matching section. The high initial value appears to be due to the instantaneously high line-charge density. The measurements are discussed in greater detail in [Fessenden et. al., 20].

\section{Concluding Remarks}

The linear induction accelerator remains a very attractive Inertial Fusion Energy driver; the recirculator offers the promise of significant cost reduction, if the technical challenges of recirculation can be met.

The LLNL Small Recirculator experiments are designed to provide a test bed for the necessary technology and to show that the beam dynamics in an induction recirculator are favorable; these experiments will pave the way to a larger-scale follow-on facility. In addition, an electron ring experiment is under development at the University of Maryland [Reiser et. al., 25, Bernal et. al., 26, and Venturini et. al., 27]. These experiments, in conjunction with the ILSE experimental program at LBNL and with detailed computer simulations, will lead to much more precise and credible predictions of heavy-ion driver behavior and cost and should allow the staged construction of the first HIF driver to begin shortly after ICF ignition is demonstrated on the National Ignition Facility. 


\section{Notes and References}

*This work was performed under the auspices of the U.S. Department of Energy by LLNL and LBNL under contracts W-7405-ENG-48 and DE-AC03-76SF00098.

[1] L. J. Laslett, Strong Focusing in Circular Particle Accelerators, in Focusing of Charged Particles, Vol. 2, A. Septier, ed., 355 (Academic Press, New York, 1967).

[2] J. J. Barnard, F. Deadrick, A. Friedman, D. P. Grote, L. V. Griffith, H. C. Kirbie, V. K. Neil, M. A. Newton, A. C. Paul, W. M. Sharp, H. D. Shay, R. O. Bangerter, A. Faltens, C. G. Fong, D. L. Judd, E. P. Lee, L. L. Reginato, S. S. Yu, and T. F Godlove, "Recirculating Induction Accelerators as Drivers for Heavy-Ion Fusion," Phys. Fluids B: Plasma Physics 5, 2698 (1993).

[3] J. J. Barnard, A. L. Brooks, J. P. Clay, E. E. Coffield, F. J. Deadrick, L. V. Griffith, A. R. Harvey, D. L. Judd, H. C. Kirbie, V. K. Neil, M. A. Newton, A. C. Paul, L. L. Reginato, G. E. Russell, W. M. Sharp, H. D. Shay, J. H. Wilson, and S. S. Yu, "Study of Recirculating Induction Accelerators as Drivers for Heavy-Ion Fusion," Lawrence Livermore National Laboratory Report UCRL-LR-108095 (1991).

[4] J. J. Barnard et. al., this volume

[5] R. O. Bangerter, "The Induction Approach to Heavy-Ion Inertial Fusion: Accelerator and Target Considerations," Il Nuovo Cimento 106 A (11), 1445-1457 (1994).

[6] H. C. Kirbie, R. L. Hanks, S. A. Hawkins, B. C. Hickman, B. S. Lee, M. A. Newton, and C. W. Ollis, "Evolution of Solid-State Induction Modulators for a Heavy-Ion Recirculator," LLNL ICF Quarterly Report for Jan.-Mar. 1995, UCRL-LR-105821-952 (1995).

[7] M. A. Newton et. al., this volume

[8] L. Reginato, M. Newton, and S. S. Yu, "Concept for Powering the Dipole Magnet in a Recirculating Induction Accelerator for Heavy Ion Fusion," Particle Accelerators 37-8, 335 (1992).

[9] D. D-M. Ho, I. Haber, K. R. Crandall, and S. T. Brandon"Octupole Correction of Geometric Aberrations for High-Current Heavy Ion Beams," Particle Accelerators 36, 141 (1991).

[10] D. Neuffer, "Geometric Aberrations in Final Focusing for Heavy Ion Fusion," Proc. of the HIF Workshop, Argonne National Laboratory, Sept. 19-26, 1978, ANL79-41, p. 333.

[11] FEAC Panel 7 Report, "Inertial Fusion Energy," prepared for the U.S. Department of Energy's Fusion Energy Advisory Committee, April 2, 1993 (unpublished).

[12] T. J. Fessenden, D. P. Grote, and W. M. Sharp, "Accelerator Waveform Synthesis and Longitudinal Beam Dynamics in a Small Induction Recirculator," Proceedings of the 1995 Particle Accelerator Conference, Dallas, TX, May 1-5, 1995 (to be published).

[13] S. M. Lund et. al., this volume

[14] W. M. Sharp, J. J. Barnard, D. P. Grote, S. M. Lund, and S. S. Yu, "Envelope Model of Beam Transport in ILSE," Proceedings of the 1993 Computational Accelerator 
Physics Conference, Pleasanton, CA, 22-26 February 1993 (AIP Conference Proceedings Vol. 297), R. D. Ryne, Ed., pp. 540-548.

[15] A. Friedman, D. P. Grote, and I. Haber, "Three-Dimensional Particle Simulation of Heavy-Ion Fusion Beams," Phys. Fluids B: Plasma Physics 4, 2203 (1992).

[16] D. A. Callahan, A. B. Langdon, A. Friedman, and I. Haber, "Longitudinal Beam Dynamics for Heavy Ion Fusion," Proc. 1993 IEEE/APS Part. Accel. Conf., IEEE Cat. No. 93 CH3279-7 p. 730 (1993).

[17] J. J. Barnard, H. D. Shay, S. S. Yu, A. Friedman, and D. P. Grote, "Emittance Growth in Heavy Ion Recirculators," Proc. 1992 Linear Accel. Conf., Ottawa, Ontario, Canada, AECL-10728, 229 (1992).

[18] D. P. Grote et. al., this volume

[19] F. J. Deadrick, J. J. Barnard, T. J. Fessenden, J. Meredith, and J. Rintamaki, "Development of Beam Position Monitors for Heavy Ion Recirculators," Proceedings of the 1995 Particle Accelerator Conference, Dallas, TX, May 1-5, 1995 (to be published).

[20] T. J. Fessenden et. al., this volume.

[21] S. Eylon, E. Henestroza, and F. J. Deadrick, "K+ Diode for the Heavy Ion LLNL Recirculator Accelerator Experiment," Proceedings of the 1995 Particle Accelerator Conference, Dallas, TX, May 1-5, 1995 (to be published).

[22] V. P. Karpenko, J. J. Barnard, F. J. Deadrick, A. Friedman, D. P. Grote, S. M. Lund, J. W. Meredith, L. A. Nattrass, M. B. Nelson, M. A. Newton, G. P. Repose, T. C. Sangster, W. M. Sharp, T. J. Fessenden, D. B. Longinotti, and C. O. Ward, "Mechanical Design of Recirculating Induction Accelerator Experiments for Heavy-Ion Fusion," Proceedings of the 1995 Particle Accelerator Conference, Dallas, TX, May 1-5, 1995 (to be published).

[23] J. J. Barnard, G. C. Caporaso, and S. S. Yu, "1-D Simulations of Heavy-Ion Injectors," Proceedings of the 1993 Particle Accelerator Conference, Washington, DC, May 17-20, 1993, Vol. 1, p. 712.

[24] M. Lampel and M. Tiefenback, "An Applied Voltage to Eliminate Current

Transients in a One-Dimensional Diode," Appl. Phys. Lett. 43, 57 (1983).

[25] M. Reiser et. al., this volume

[26] S. Bernal et. al., this volume

[27] M. Venturini et. al., this volume

"This work was performed under the auspices of the U.S. Department of Energy by Lawrence Livermore National Laboratory under contract No. W-7405-Eng-48. 


\section{Figure Captions}

Figure 1. Artist's rendering of recirculator-driven power plant.

Figure 2. Final configuration of the Small Recirculator.

Figure 3. Insertion/extraction section.

Figure 4. One-quarter of the Small Recirculator (CAD rendering).

Figure 5. Two half-lattice periods of the Small Recirculator (CAD rendering). The top of the left-hand half-lattice period is cut away to reveal the internal structure.

Figure 6. Linear configuration (length $4.568 \mathrm{~m}$ from source to final Faraday cup).

Figure 7. Interior of source tank, showing back of source and (at left) hole through which beam passes on its way into matching section. Holes of varying size can be used.

Figure 8. Straight transport section of the Small Recirculator. Each spool holds two permanent-magnet quadrupoles; the entire section uses seven quadrupoles. The large enclosure at the left foreground contains diagnostic apparatus. Orientation is opposite to that of Fig. 8.

Figure 9. Current at the Faraday cup $1.9 \mathrm{~m}$ downstream of the diode source, and corresponding results from the 1-D code HINJ.

Figure 10. Measurement of horizontal normalized emittance at end of matching section. 


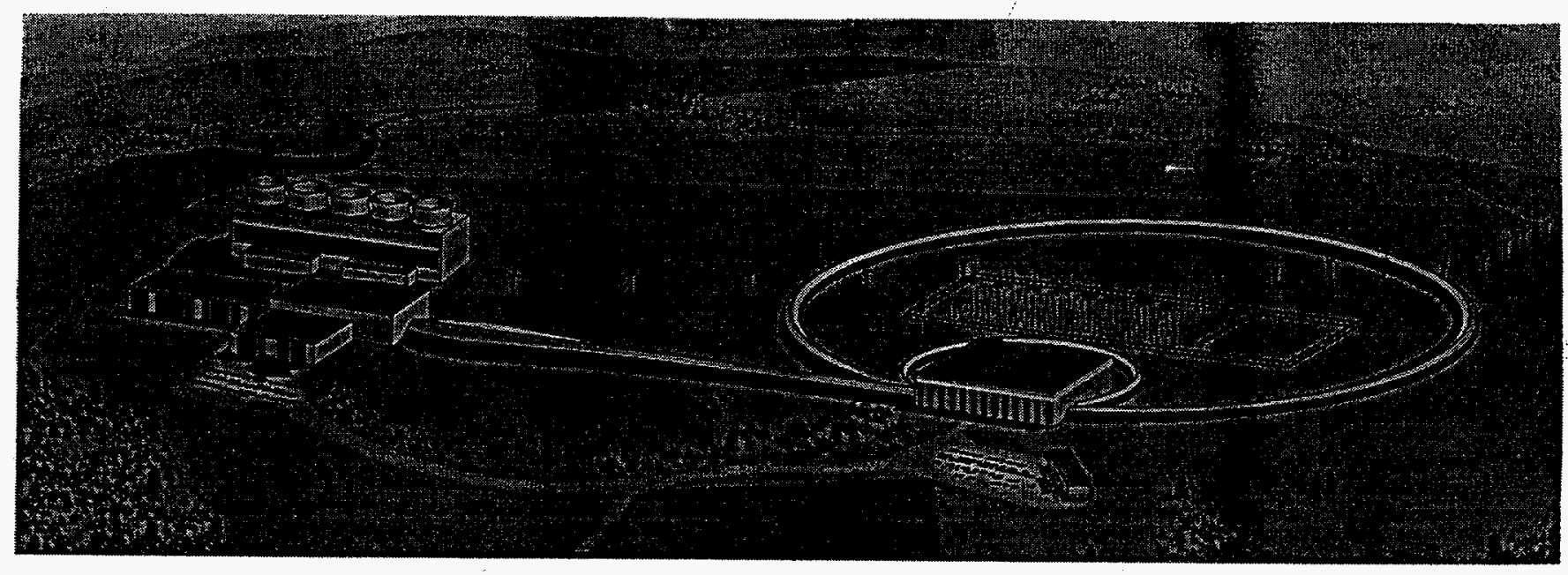

Friedman 1 


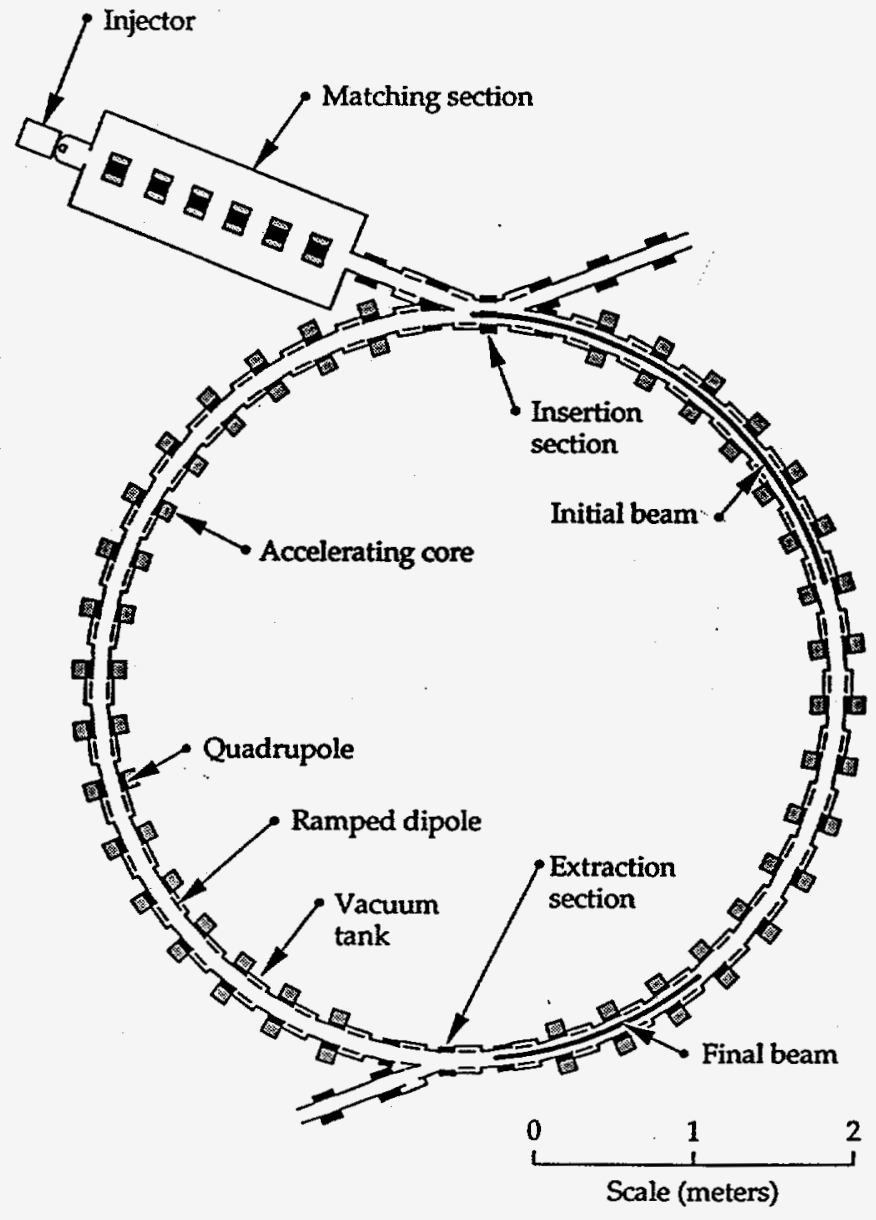

FRIEDMAN 2 


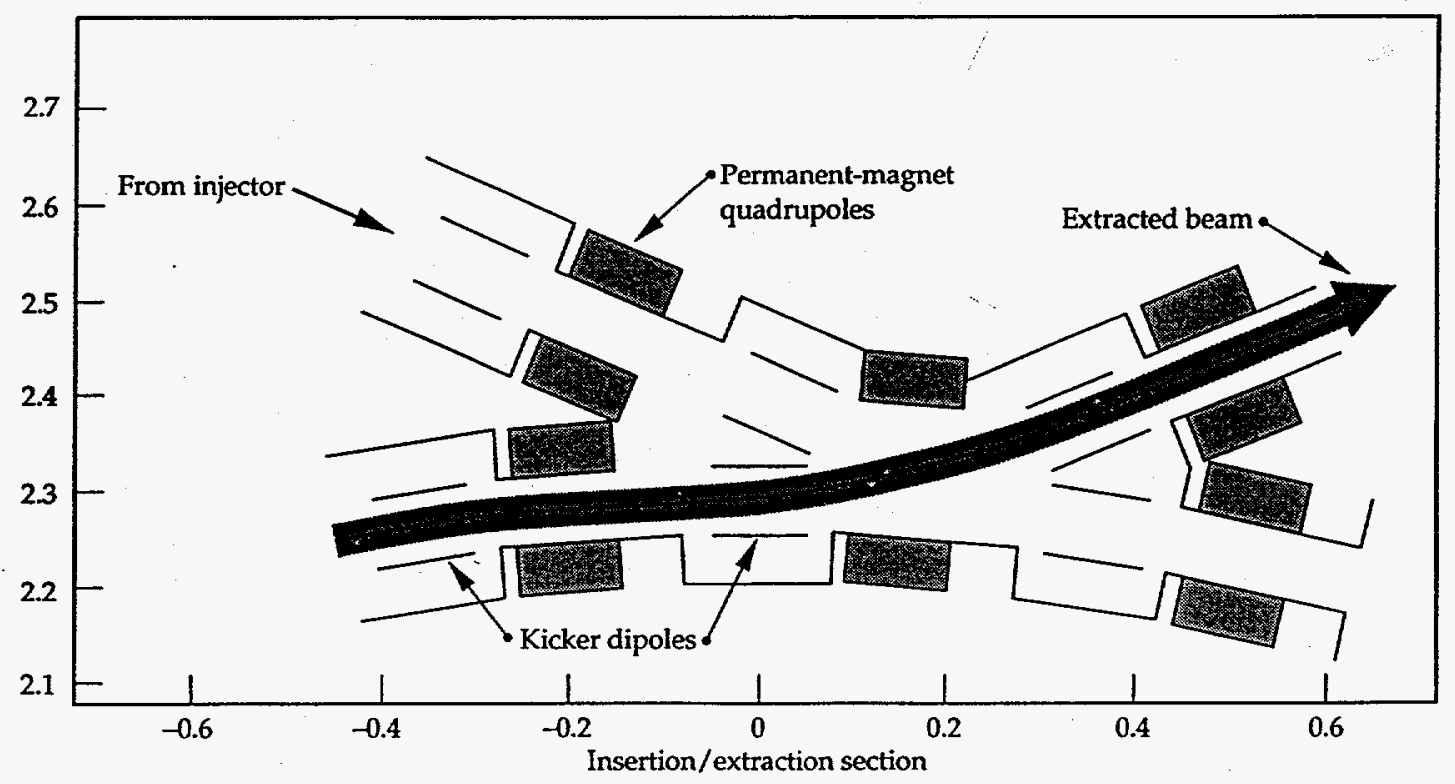

FriedunA 3 


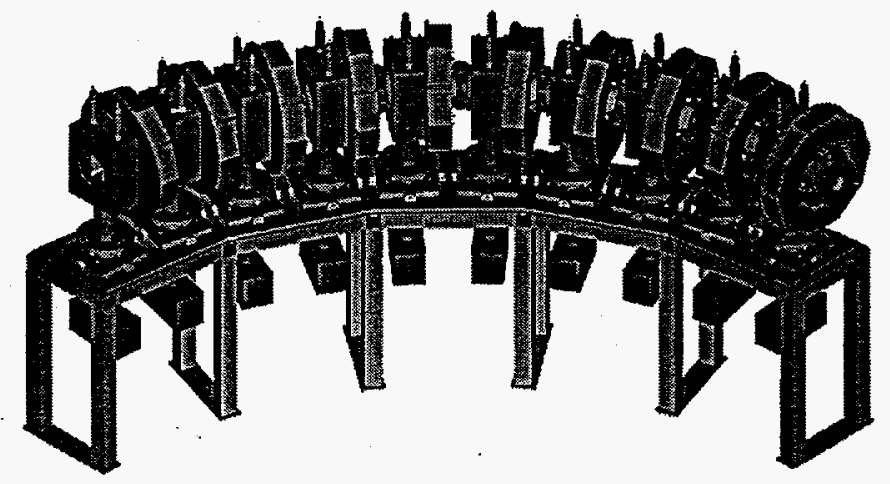

FRIEDMAN 4 


$$
S \text { Nmotid }
$$

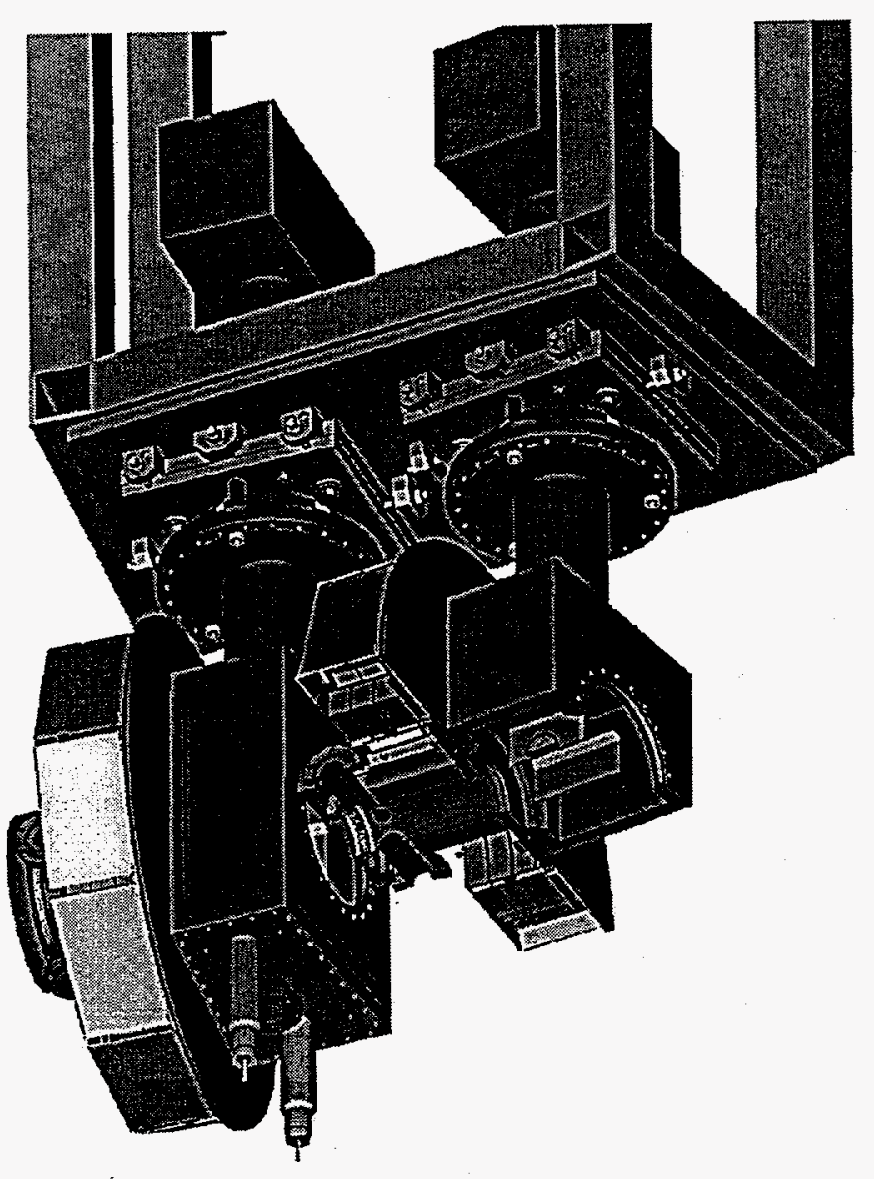




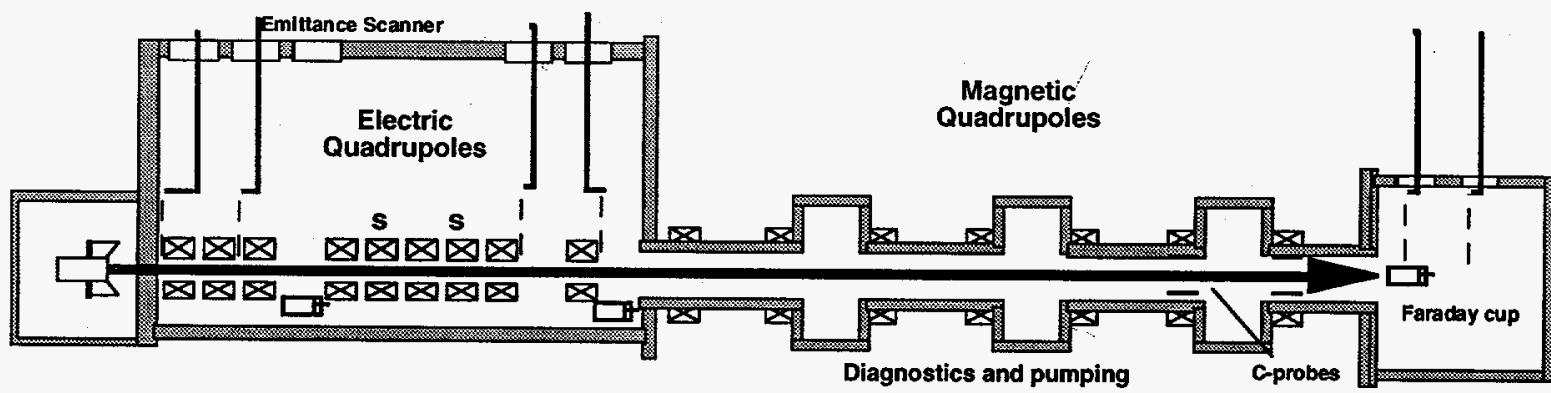

Injector Matching section

Drift section

FRIEDMAN 6 


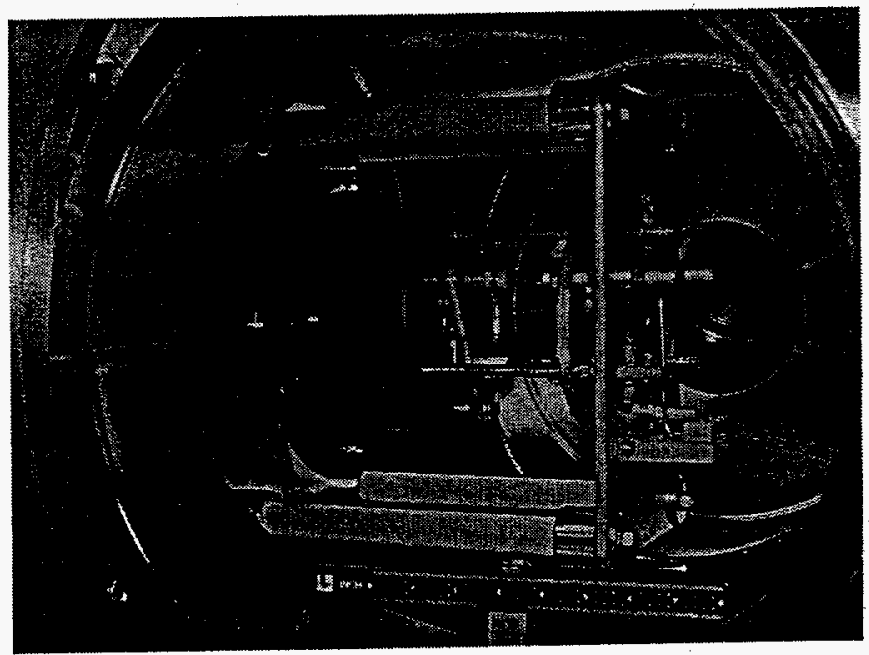

FreDuan 7 


$$
8 \text { Nimcary }
$$

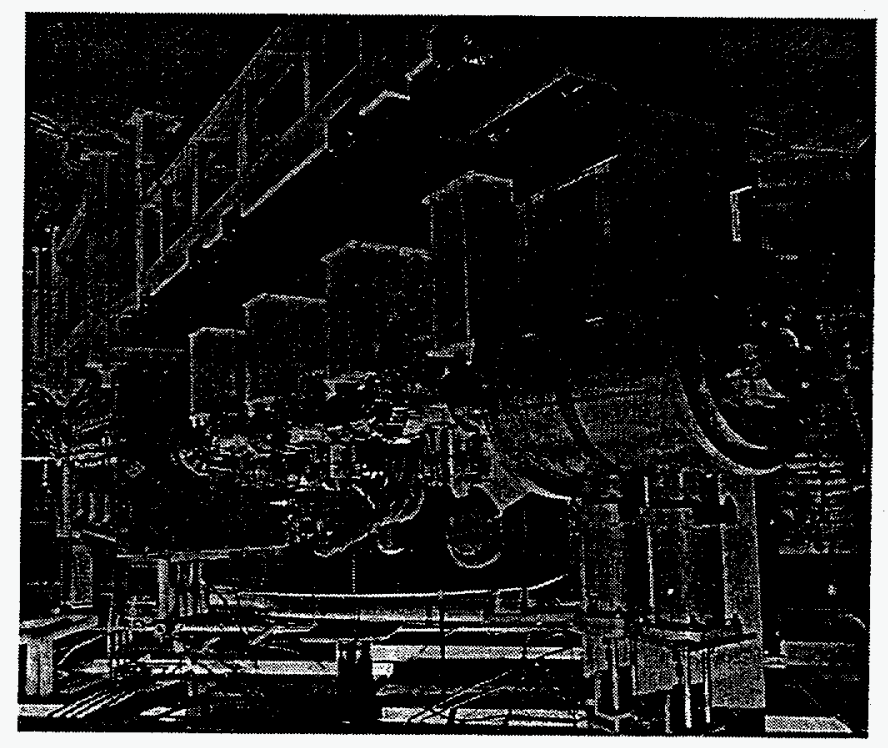




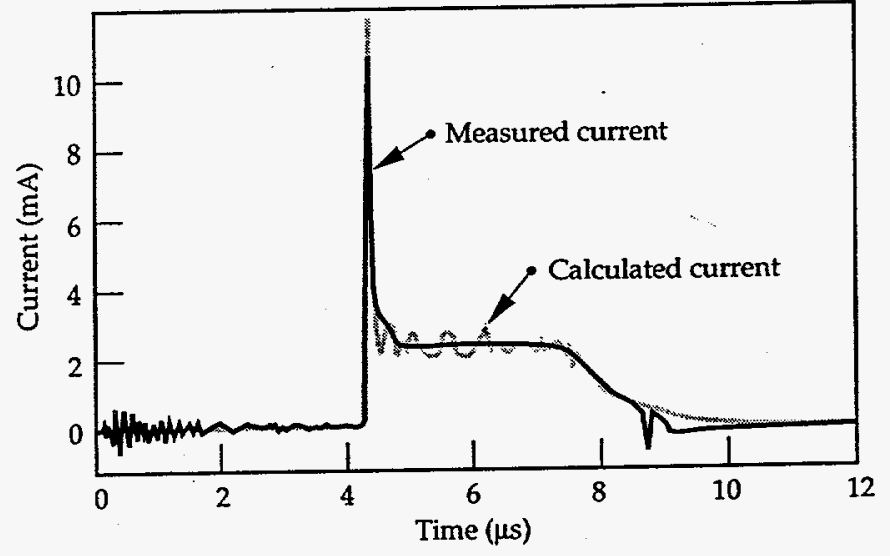

FRIEDMUAN 9 


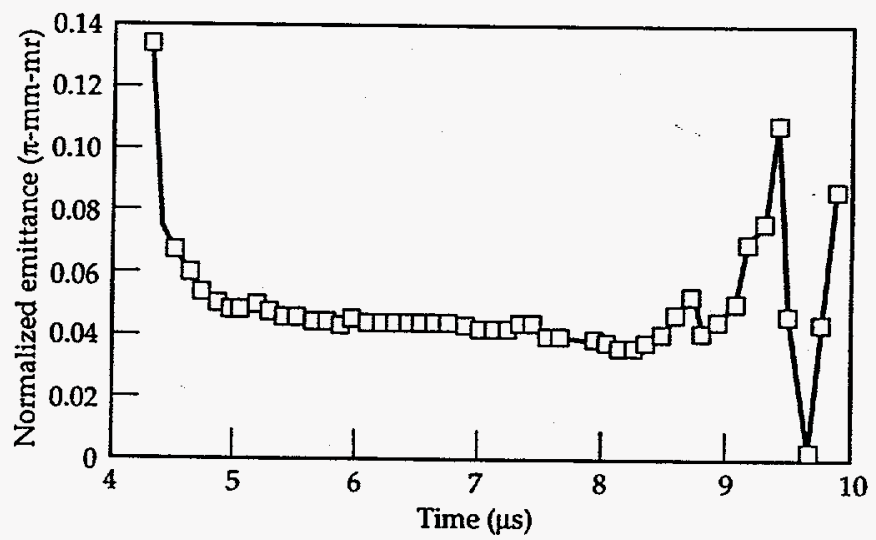

Frisiduar 10 